\title{
Effect of Cross Linking on the Toxicity of CdTe QDs in Solid/ Liquid Medium
}

\author{
Poonam Bichpuria $^{{ }^{*}}$, Anjali Oudhia ${ }^{2}$,Pragya Kulkarni ${ }^{3}$ \\ ${ }^{I}$ (Department of Physics, Govt.V.Y.T.PG Autonomous College Durg (C.G.), G.E. Road, Durg (C.G.), India, \\ Pin code-491001) \\ ${ }_{2}^{2}$ (Department of Physics, Govt.Nagarjun Autonomous College of Science,Raipur (C.G.), India, Pin code- \\ 492001) \\ ${ }_{3}^{3}$ (Department of Microbiology, Govt.V.Y.T.PG Autonomous College Durg (C.G.), G.E. Road, Durg (C.G.),India, \\ Pin code-491001)
}

\begin{abstract}
Highly luminescent GSH capped CdTe quantum dots (GSH-CdTe QDs) were prepared through a facile microwave $(M W)$ aided wet chemical method. The GSH-CdTe QDs were water soluble and biocompatible. The higher intensity of Photoluminescence (PL) emission can be attributed to the highly crystalline structures of crosslinked GSH-CdTe quantum dots (cGSH-CdTe QDs) observed in the X-ray diffraction (XRD).The SEM micrograph shows densely populated colony of CdTe QDs with almost spherical morphology. The cell death experiment shows that cross linking effectively controls the toxicity of CdTe QDs both in liquid and solid media which makes them suitable for biomedical application. Toxicity control was attributed to the effective surface passivation through cross-linking the GSH shell with 1-ethyl-3-(3-dimethyl aminopropylcarbodiimide hydrochloride/N-hydroxysuccinimide (EDC/NHS).
\end{abstract}

Keywords: Microwave (MW), Photoluminescence (PL), Toxicity, X-ray diffraction (XRD).

\section{Introduction}

Nanostructured materials [1] are of importance because they can link the gap between the bulk and molecular levels and lead to exclusively new avenue for applications, particularly in electronics, optoelectronics and biology. Nanomaterials are being used in removal of harmful micro-organisms,recognition and treatment of various diseases[2].The formation of nanoparticles changes the properties of many conventional materials e.g., the larger surface area of nanoparticles can result in a greater degree of interaction with bacterial cell walls[3].Colloidal II-VI semiconductor nanocrystals or quantum dots (QDs) have attained a great research focus due to their advantages in optical properties including tunable emission spectra, high photostability, resistance to photobleaching and controllable surface characteristics. These properties have broadened the range of applications of QDs in advance studies like protein trafficking [4,5] and in the treatment of cancer [6,7]. For biological and environmental applications, it is essential to prepare non toxicCdTe QDs in Toluene directly. In contrast to organometallic techniques, solution growth method appears to be a safer, low cost and more convenient approach because it does not involve special instrumentation, poisonous intermediates, and the growing-rate could be easily controlled. The GSH-CdTe QDs show similar optical properties as QDs synthesized using more complicated and expensive methods. Further, a decreased level of $\mathrm{Cd}^{2+}$ ions and higher amounts of GSH was effectively used to lower the toxicity of the as prepared CdTe QDs as compared to QDs produced by other chemical methods [8].Further, the CdTe nanoparticles obtained by the present method were spherical; making them well- suited for the bio medical application.

\subsection{Synthesis of GSH-CdTe QDs}

\section{Materials and Methods}

$182 \mathrm{mg} \mathrm{CdCl} 2 \cdot 2.5 \mathrm{H}_{2} \mathrm{O}$ was diluted with $40 \mathrm{ml}$ of Toluene. During the constant stirring $240 \mathrm{mg}$ GSH, $10 \mathrm{mg}$ Sodium citrate, $2 \mathrm{ml}$ of $\mathrm{K}_{2} \mathrm{TeO}_{3}$ sol and $20 \mathrm{mg}$ of $\mathrm{NaBH}_{4}$ were added into Cadmium sol. $1 \mathrm{M} \mathrm{NaOH}$ was then used to adjust the $\mathrm{pH} 10$ under vigorous stirring. The mixture was kept in $\mathrm{MW}$ for $1 \mathrm{~min}$ at $300 \mathrm{~W}$. The mixture was cooled down to $\sim 50^{\circ} \mathrm{C}$. The as prepared CdTe solution concentrated to $14^{\mathrm{Th}}$ of the original volume was precipitated using 2-propanol and collected via centrifugation. CdTe QDs dispersion was prepared by redissolving this colloid precipitate in $3 \mathrm{ml}$ DD water.

\subsection{Cross linking of GSH-CdTe QDs (cGSH-CdTe QDs)}

For cross linking; $0.5 \mathrm{ml}$ of GSH-CdTe QDs were suspended in water $(10 \mathrm{mg} / \mathrm{ml})$ and dissolved with 50 $\mathrm{ml}$ of DMSO (Dimethylsulfoxide).Then 600mg NHS and $4 \mathrm{ml}$ of EDC were added with stirring and reacted for $30 \mathrm{~min}$ atroom temperature. After that $300 \mathrm{mg} \mathrm{GSH}$ dissolved in $5 \mathrm{ml}$ of water and added drop wise to QDs solution. After overnight reaction cGSH-QDs were precipitated with $100 \mathrm{ml}$ acetone by centrifugation. The supernatant was decanted and powder was re-suspended in $50 \mathrm{ml}$ of Borate buffer $(\mathrm{pH} 8.0)$ and filtered. This 
process was repeated 3 times to remove all excess reagents and GSH. Purified cGSH-QDs were re-suspended in water and filtered.

\subsection{Toxicity Study}

\subsubsection{Test Microorganism}

For studying toxicity of QDs a pure culture of E.coli was obtained which was isolated indigenously from sewage sample and identified biochemically. It was maintained on nutrient agar slants at $4^{0} \mathrm{C}$ till use.

\subsubsection{Culture Media}

Nutrient Agar (NAM pH-7.0) was prepared by dissolving $1.25 \mathrm{gm}$ of peptone, $1.25 \mathrm{gm}$ of sodium chloride, $75 \mathrm{gm}$ of beef extract and $5 \mathrm{gm}$ of agar in $250 \mathrm{ml}$ of distilled water. All contents were mixed thoroughly on a shaker. After mixing, it was sterilized by autoclaving at $121^{\circ} \mathrm{C}$ for 15 minutes. Chemicals used in all tests were of analytical quality. Deionised water was used to prepare all microbiological media.

\subsubsection{Well diffusion Method}

Toxicity activity of prepared cGSH-CdTe QDs in solid media was assayed using standard well diffusion method. Pure culture of the test bacteria was inoculated on NA plates by spread plate techniques. With a sterile borer $2 \mathrm{~mm}$ holes were punched in the medium and $15 \mu \mathrm{l}$ of the test solutions containing nanoparticles were added and the plates were incubated at $37^{\circ} \mathrm{C}$ for $24 \mathrm{~h}$.Observations were recorded for presence of zone of inhibition and their size was also measured. Sterile distilled water was used as control and three replicates were maintained for each test.

\subsection{Structure and Morphology}

\section{Result and Discussions}

The SEM micrograph of the as obtained QDs arerepresenting GSH-CdTe QDsshown in Fig.1. This micrograph shows densely populated colony of CdTe QDs with almost spherical morphology.

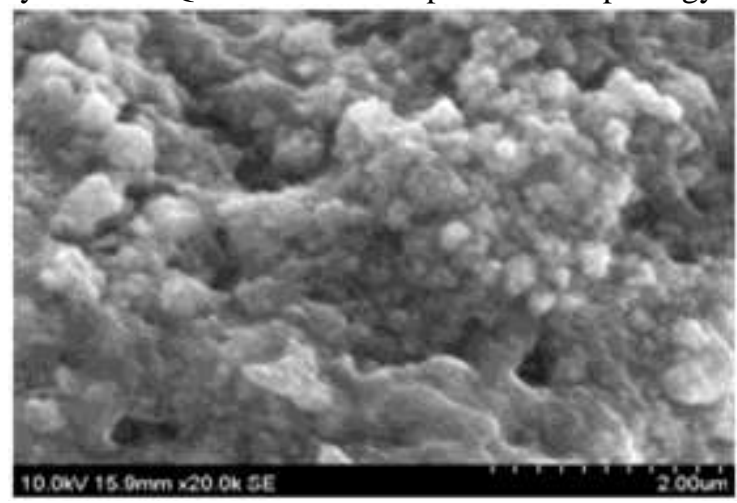

Fig.1. SEM micrograph of CdTe QDs

In Fig.2 the XRD pattern shows uncapped and capped/cross linked CdTe QDs. The XRD shows a striking change in the structure of QDs after capping shown in Fig. 2(b). The uncapped CdTe QDs show a cubic crystal structure (Fig. 2(a)), whereas the GSH capped QDs shows well defined high intensity peaks of hexagonal structure (Fig. 2(b)). This can be attributed to the presence of sulfur obtained from dissolution of GSH forming CdS layer over the CdTe QDs having a prevalent hexagonal structure $[9,10]$
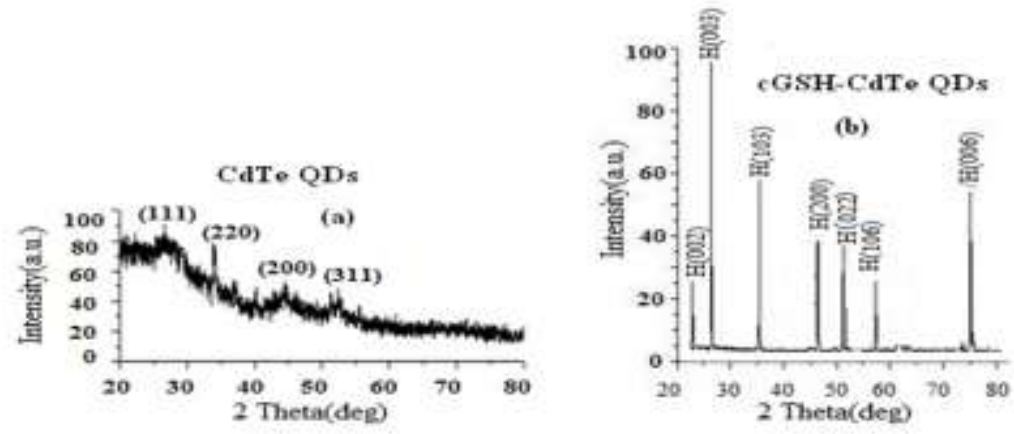

Fig. 2. XRD pattern of (a) uncapped un cross linked pure CdTe QDs showing purely cubic structure according to JCPDS No 75-2083 (b) Capped and cross linked cGSH-CdTe QDs showing a purely hexagonal structure according to JCPDS No 80-0089 


\subsection{Mechanism and effect of Cross linking on the toxicity of CdTe QDs}

The study of toxicity is of uttermost importance for the synthesis of nanocrystals for biomedical or photonic applications. In this study facile solvent, capping agents and fixation of capping agents using cross linking was adopted to synthesize CdTe QDs for bio medical applications. We used EDC as effective water soluble cross linking agent belonging to the group of carbodiimide compounds which enables peptides and proteins to be conjugated not only to one another but also to any compounds or solid surface that bear carboxyl or amino group.One of the most commonly used bioconjugation methods is offered by a zero-length cross linking agent EDC to conjugate carboxylic acid functional QDs and primary amine-containing biomolecules [11]. The mechanism of cross linking is shown in Fig. 3.The QDs firstly react with EDC and forms an aminereactive O-acylisoureaintermediate. To stabilize this intermediate, sulfo-NHS is added to form a more stable form of the amine-reactive sulfo-NHS ester. With the present of amine-containing biomolecules, QDs are finally conjugated with biomolecules with a stable amide bond. The major advantage of this method is that proteins generally contain primary amine groups and therefore no pretreatment of proteins is needed. However as a drawback, conjugation can occur to different bonding sites randomly on the protein.

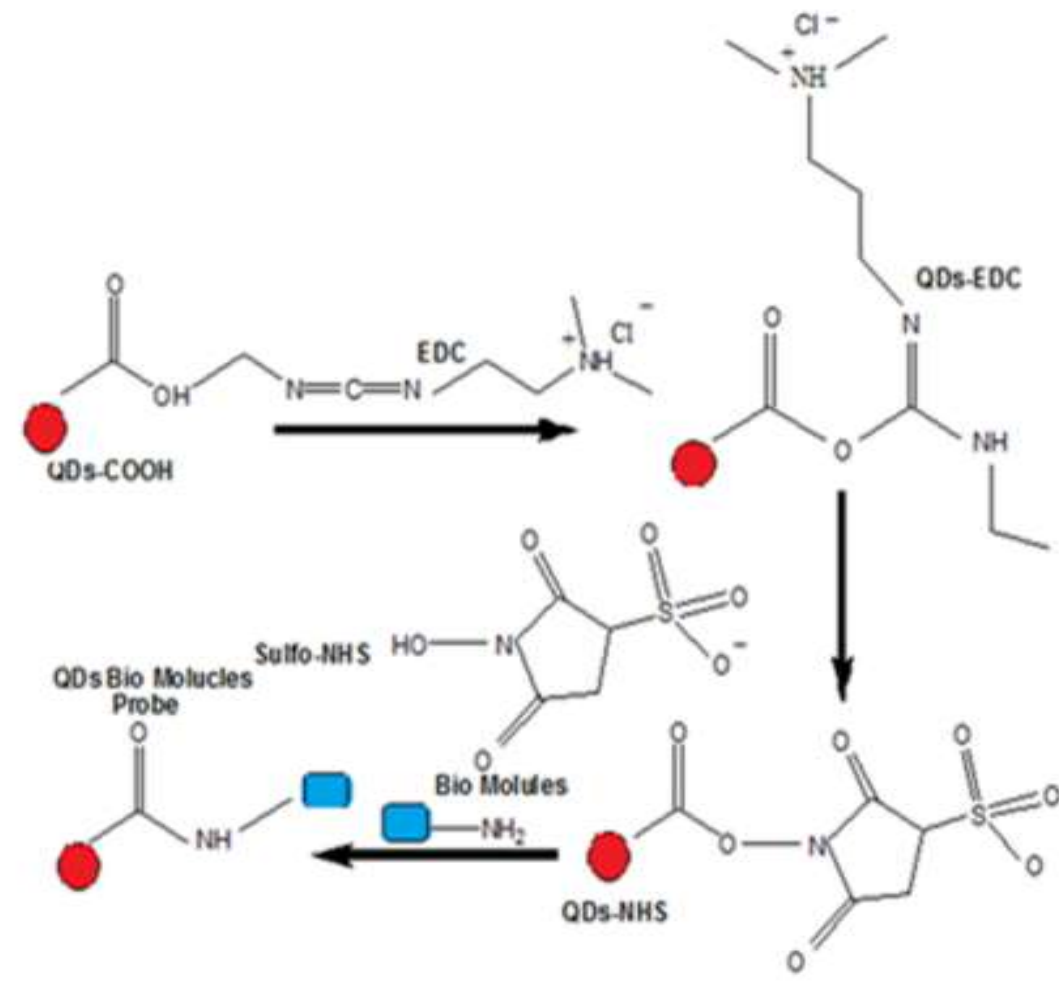

Fig. 3. Schematic diagram showing reactions of conjugating carboxyl-QDs to amine-containing biomolecules.

\subsection{Toxicity Test}

Experiment conducted under the solid media, reveals that CdTe QDs show toxicity for the bacteria used under study (Fig. 4). Toxicity was measured by comparing zone of inhibition after 48 and $72 \mathrm{hrs}$. Un crosslinked GSH-CdTe shows an increase in toxicity with time in this case (Fig. 4(a)), however, cGSH-CdTe shows negative result for toxicity and no zone of inhibition was observed in Fig. 4(b). The bacteria grown in a liquid media were exposed to the QDs under submerge condition for testing the toxicity of QDs in liquid medium and the toxicity was observed in terms of turbidity as compared to control (pure water). Un cross-linked GSH-CdTe QDs showed toxicity with time, as the turbidity was decreased (optical density increased) over the control, showing death of bacterial colony. However, even after 72 hrs, cGSH-CdTe QDs show no decrease in turbidity and hence no toxicity. This shows that cross linking effectively controls toxicity in liquid media too. 


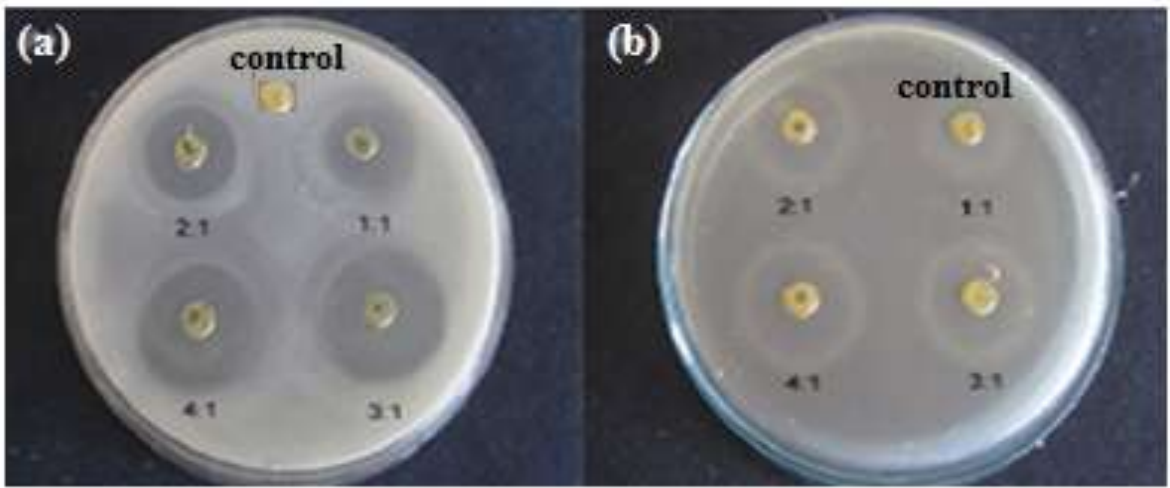

Fig. 4. Cytotoxicity test in solid media (a) GSH-CdTe QDs showing smaller zone of inhibition after 48 hrs and a larger circle showing growth of the zone of inhibition after 72 hrs. (b) cGSH-CdTeQDs showing no zone of inhibition even after $72 \mathrm{hrs}$.

(a)

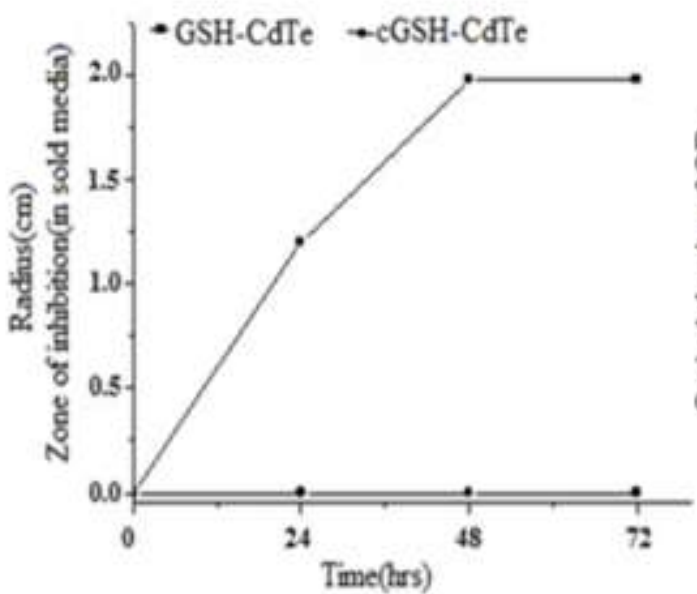

(b)

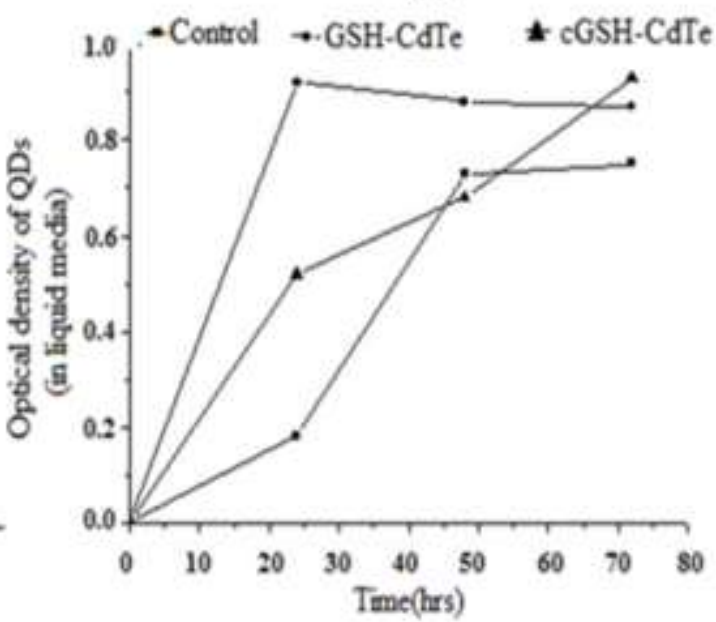

Fig. 5. Cytotoxicity test of GSH-CdTe and cGSH- CdTe QDs (a) zone of inhibition not observed with time for cGSH- CdTe QDs (In solid media) showing non toxicity (b) increasing optical density in terms of turbidity for cGSH-CdTe proves its non toxicity by showing growth of bacterial colony (in liquid media).

The results of toxicity test in solid media shown in Fig. 5(a). The flat line shows absence of zone of inhibition in cGSH-CdTe QDs as compared to GSH-CdTe for which an increase in the zone of inhibition which gradually becomes constant with time. While it can be observed that the optical densities (measured in terms of turbidity) of the cGSH-CdTe is comparable with that observed in control, showing increasing turbidity due to bacterial growth ie absence of toxicity, after cross linking, whereas the optical densities in case of GSH-CdTe did not change at all showing no growth in the bacterial colonies due to toxicity (Fig. 5(b)).

\subsection{Effect of cross linking on the PL of CdTe QDs}

The PL emission spectra of CdTe QDs shown in Fig. 6.The PL emission spectra were obtained using Photoluminescence spectrophotometer.The PL emission peak for CdTe was centered at $520 \mathrm{~nm}$ for uncapped CdTe QDs, at $500 \mathrm{~nm}$ for GSH-CdTe and at $460 \mathrm{~nm}$ for cGSH-CdTe. Firstly, the PL peaks obtained for CdTe in our case are blue shifted as compared to bulk CdTe. A strong quantum confinement regime can be one reason for the blue shift in case of pure CdTe QDs. In case of cGSH-CdTe the PL emission peak intensity is enhanced further with a blue shift as compared to both CdTe and GSH-CdTe QDs. 


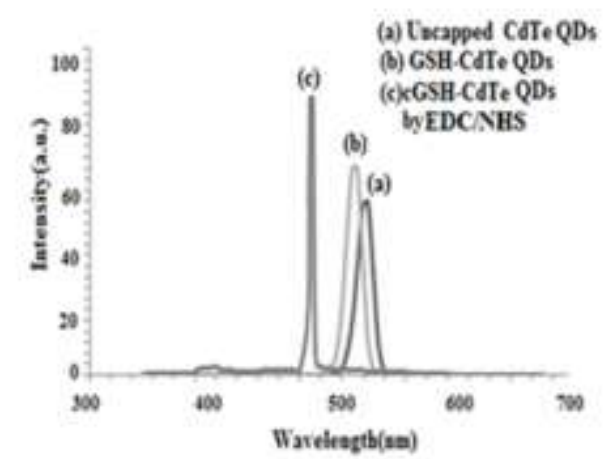

Fig. 6.PL emission spectra of (a) uncapped CdTe QDs (b) GSH-CdTe QDs. (c) cGSH-CdTe QDs cross linked by EDC/NHS.

The higher intensity of PL emission can be attributed to the highly crystalline hexagonal structures of cGSH-CdTe observed in the XRD pattern(Fig. 2(b)) as compared to that of pure CdTe QDs, which show amorphous like cubic structures(Fig. 2(a))[12]. High density peptides can be achieved through the EDC cross linking process as it also leads to their polymerization linking carboxyl-to-amine. Addition of NHS increases the efficiency of this process. These features help not only in effective surface passivation and adhesion, reducing toxicity, but also enhance PL intensities. Further the polymerization property of EDC/NHS leads to a dense shell formation of polymeric GSH wrapping CdTe QDs even more tightly, which may reduce the non- radiative channels further, leading to a blue shift.

\section{Conclusions}

Present study can be used to achieve effective control the toxicity of CdTe QDs for biomedical applications. Toxicity control in solid as well as liquid media using surface passivation and cross-linking was achieved. A green and cost effective approach for producing high quality non toxic QDs makes this study even more useful.

\section{Acknowledgements}

We are highly grateful to Dr. P. Kulkarni, Assistant Professor,Dept. of Microbiology at Govt. V.Y. T. PG Autonomous College, Durg (C.G.) for the Cytotoxicity experiment. We acknowledge the kind help and support of Dr.S.J.Dhoble Associate Professor, Dept. of Physics at RTM Nagpur University for PL studies, Dr. D. M. Phase scientist-G and V.K. Ahire at CSIR Indore for SEM studies and N. Kulkarni at TIFR (Mumbai) for XRD studies.

\section{References}

[1]. D.A. Higgins, S. C. Park, Khanh-Hoa Tran-Ba, and T. Ito, Single-Molecule Investigations of Morphology and Mass Transport Dynamics in Nanostructured Materials, Annu. Rev. Anal. Chem. 8, 2015,193-216.

[2]. P.C.Nagajyoti, T.N.Prasad,V.K.V. Kapduklee,V.M.Kapduklee, Bio-Fabrication Of Silver Nanoparticles Using Leaf Extract Of SaururusChinenis,Dig.j.Nano.Biostru.6, 2011, 121-133.

[3]. M. Shah, D. Fawcett , S. Sharma, S Kumar Tripathy and G. Eddy Jai Poinern, Green Synthesis of Metallic Nanoparticles via Biological Entities, Mater. 8, 2015,7278-7308.

[4]. C. D. Valentine, A.S. Verkman, and P. M. Haggie, Protein trafficking rates assessed by quantum dot quenching with bromocresol green, Traffic,13, 2012,25-28.

[5]. C.D. Valentine, P.M. Haggie, Confinement of $\beta 1$ and $\beta 2$-adrenergic receptors is the plasma membrane of cardiomyocyte-like H9c2 cells is mediated by selective interactions with PDZ domain and Akinase anchoring proteins but not caveolae, Mol. Biol. Cell. 22,2011,2970-2982.

[6]. S. B. Rizvi, ShirinGhaderi, Mo Keshtgar,and A. M. Seifalian, Semiconductor quantum dots as fluorescent probes for in vitro and in vivo bio-molecular and cellular imaging ,Nano Rev.1, 2010,1-15

[7]. M. Fang, Chun-weiPeng, Dai-Wen Pang, and Y. Li, Quantum Dots for Cancer Research: Current Status, Remaining Issues, and Future Perspectives,Cancer Biol. Med.9,2012, 151-163.

[8]. J.M. Perez-Donoso, J.P. Monra's, D. Bravo, A. Aguirre, A.F.Quest,et al., Biomimetic, Mild Chemical Synthesis of CdTe-GSH Quantum Dots with Improved Biocompatibility, PLoS ONE 7(1) e30741.doi:10.1371/journal. pone.0030741, 2012.

[9]. M. Pal, N. R. Mathews, P.Santiago and X. Mathew, A facile one-pot synthesis of highly luminescent CdS nanoparticles using thioglycerol as capping agent, J. Nanopart Res. 14,2012, 1-13.

[10]. J.Wang, Y.Long, Y.Zhang, X.Zhong and L.Zhu, Preparation of highly luminescentCdTe/CdS core/shell quantum dots, Chem. Phys. Chem.9,2009, 680-685.

[11]. J.Barar and Y.Omidi, Surface modified multifunctional nanomedicines for simultaneous imaging and therapy of cancer,Bioimpac. 4,2014, 3-14.

[12]. A.V. Kokate, M.R. Asabe, P.P. Hankare and B.K. Chougule, Effect of annealing on properties of electrochemically deposited CdTe thin films, J. Phys. Chem. Solids. 68 ,2007, 53-58. 